\title{
A New Visualization Method for Virtual Colonoscopy
}

\author{
F.M. Vos ${ }^{1,2}$, I.W.O. Serlie ${ }^{1,3}$, R.E. van Gelder ${ }^{4}$, F.H. Post ${ }^{3}$, \\ R. Truyen ${ }^{5}$, F.A. Gerritsen ${ }^{5}$, J. Stoker ${ }^{4}$, A.M. Vossepoel ${ }^{1}$ \\ ${ }^{1}$ Pattern Recognition Group, Delft University of Technology \\ Lorentzweg 1, 2628 CJ Delft, The Netherlands \\ \{frans, iwo, albert\}@ph.tn.tudelft.nl \\ ${ }^{2}$ Department of Radiology, University Hospital Rotterdam \\ Dr. Molewaterplein 40, 3015 GD Rotterdam, The Netherlands \\ frans@ph.tn.tudelft.nl \\ ${ }^{3}$ Computer Graphics and CAD/CAM Group, Delft University of Technology \\ Zuidplantsoen 4, 2628 BJ Delft, The Netherlands \\ frits.post@cs.tudelft.nl \\ ${ }^{4}$ Department of Radiology, Academic Medical Centre Amsterdam \\ P.O. Box 22700, 1100 DE Amsterdam, The Netherlands \\ r.e.vangelder@amc.uva.nl \\ ${ }^{5}$ EasyVision Advanced Development Group, Philips Medical Systems Nederland B.V. \\ P.O. Box 10000, 5680 DA Best, The Netherlands \\ \{roel.truyen, frans.gerritsen\}@philips.com
}

\begin{abstract}
Virtual colonoscopy or 'colonography' is a patient-friendly, modern screening technique for polyps. Automatic detection of polyps can serve to assist the radiologist. This paper presents a method based on clustering the principal curvatures. Via automatic polyp detection $5 / 6$ polyps $(>5 \mathrm{~mm})$ were detected at the expense of 9 false positive findings per case. For visualization, the bowel surface is presented to the physician in a 'panoramic' way as a sequence of unfolded cubes. Conventionally, only $93 \%$ of the colon surface is available for examination. In our approach the area in view is increased to $99.8 \%$. The unfolded cube visualization is another step to optimize polyp detection by visual examination. Experiments show a sensitivity of $10 / 10$ (on a per patient basis) for any polyp. The specificity was $7 / 10$.
\end{abstract}

Keywords: radius of curvature, scientific visualization, virtual endoscopy.

\section{Introduction}

Colorectal polyps are considered important precursor of colon cancer [1]. Typically, such a benign tumour presents a sphere extending from the bowel wall on a small, thin stem (like a mushroom, see Figure 1). It may be distinguished from the physiologic surface structure by its isotropic curvature in the protruding direction (opposite to its surroundings). Commonly, only polyps larger than $5 \mathrm{~mm}$ are considered significant. This minimum polyp size is justified by a strong correlation between its size and the risk of 
a malignant transformation [2]. Smaller polyps are nearly always benign, in which case an operation is unnecessary [3]. Not surprisingly, early detection of polyps has proven to lead to a decrease in incidence of colon cancer [4]. The interval before polyps develop into a malignancy is estimated to be in the order of 5 years [2]. Hence, screening seems an attractive possibility for prevention.

Until recently a barium enema and camera colonoscopy were the two procedures available for examining the colon [5][6]. Both techniques have serious drawbacks. The sensitivity of the barium enema is only $50-80 \%$ for polyps sized from 5 to $10 \mathrm{~mm}$ in diameter. Camera colonoscopy requires intravenous sedation to ease the discomfort. Consequently, many of those eligible for screening avoid the examination [7].

In the past few years, virtual colonoscopy ('colonography') has been developed as a modern alternative [8]. Generally, the procedure comprises of the following steps. First, the patient's colon is cleansed and distended by transanal inflation with air. Subsequently, a 3D image volume is acquired of the abdomen by CT or MRI. Finally, the interior bowel surface is extracted and visualized, after which the physician virtually navigates through the colon and examines the surface for abnormalities (Figure 1, left).

Virtual colonoscopy relies heavily on a proper representation of geometric information. Important clues such as vascularization and wall texture are unavailable. Some authors suggest to support the exploitation of surface shape by automatic polyp detection. As yet, only preliminary results have been published, most in medical journals and not specifically focussed on technical details. Such detection was based on classification of the principal curvatures, local sphere fitting and orientation clustering of surface normals [9][10]. Although the development is still in an early stage, it is well recognized that automatic polyp detection may enhance the efficiency of the examination.

The current procedure is to explore the bowel in an interactive way. However, real-time visualization at a high frame-rate is not always possible due to the large data size and the inherent quality requirements. The latter problem is often solved by generating movies off-line. Typically, sequences are generated, with forward and backward viewing directions. In such a way, the observed part of the surface is maximized. Still, selective images on the colon wall are obtained. It may well be that important parts of the surface are missed, while insignificant parts are reviewed twice (Figure 1, right).

For a practical application, polyp detection must comply with requirements regarding

- scale dependency

- sensitivity/specificity

- effectiveness (in terms of area inspected)

The calculation of image features often introduces a scale parameter (e.g. the width of Gaussian derivatives). Special care must be taken to control the scale dependency of the outcome. In any case there must be a high sensitivity for detecting polyps larger than $0.5 \mathrm{~cm}$ in diameter, without introducing too many false positives. The previous claim is supported by making the complete surface accessible for inspection (hence the conventional dual sided view).

In this paper we will present a novel technique to detect polyps which comprises three steps: 

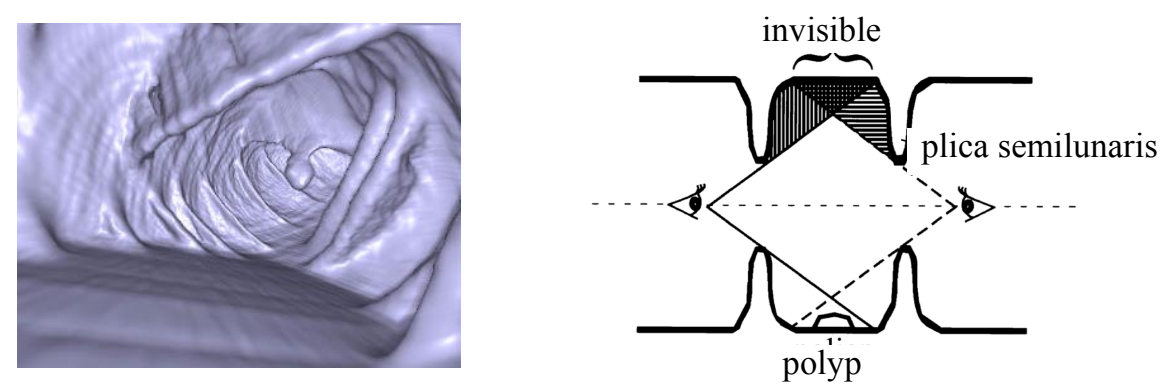

Fig. 1. Colonographic view on a polyp (left). The right picture shows which urface parts missed in two sided views.

- calculation of the principal isophote radii of curvature

- clustering

- visualization

The use of curvature information derives from the characteristic shape of polyps (see above). However, direct classification of the curvatures is insufficient for efficient detection (by yielding too many false positives). For that reason we introduced a clustering step to identify significant polyps and reject erroneous findings. The results serve to support the radiologist while he explores virtual endoscopic data. To that end, we integrated our method in a visualization environment presenting the bowel surface physician in a 'panoramic' way.

The paper is organized as follows. In Section 2, we will methodically describe the three stages of the technique. Results including a clinical evaluation will be presented in Section 3. The paper will finish by drawing conclusions and describe suggestions for future work.

\section{Technique}

\subsection{Calculation of the Principal Normal Curvatures}

A three step algorithm to calculate the principal curvatures from a 3D image volume is as follows [11]:

- Step 1

Calculate the gradient vector $(g)$ and the Hessian matrix $(H)$ in each position. They are defined as:

$$
g=\left(I_{x}, I_{y}, I_{z}\right) \quad H=\left[\begin{array}{ccc}
I_{x x} & I_{x y} & I_{x z} \\
I_{x y} & I_{y y} & I_{y z} \\
I_{x z} & I_{y z} & I_{z z}
\end{array}\right]
$$

where each entry represents a partial derivative of the 3D image $I$. Each derivative is calculated through convolution with a Gaussian kernel at a specific width $\sigma_{\text {Gauss. }}$ 
- Step 2

Rotate the Hessian matrix so that one axis aligns with the gradient direction. The resulting matrix $H^{\prime}$ 'can be written as:

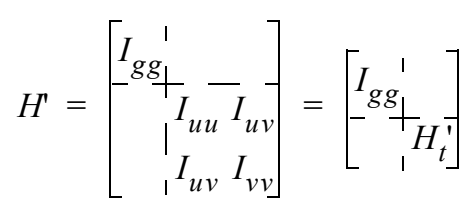

Here, $I_{g g}$ is the second derivative in the gradient direction and $H_{\mathrm{t}}^{\prime}$ a $2 \mathrm{D}$ Hessian in the tangent plane perpendicular to $g$.

- Step 3

Compute the eigenvalues of $H_{\mathrm{t}}^{\prime}$. These values $\lambda_{1}$ and $\lambda_{2}$ are respectively the maximum and the minimum second derivatives in the touching plane. The corresponding eigenvectors point in the direction of maximal and minimal curvature. It can be shown that the principal radii of curvatures are given by

$$
\kappa_{1}=\frac{\|g\|}{-\lambda_{1}} \quad \kappa_{2}=\frac{\|g\|}{-\lambda_{2}}
$$

\subsection{Clustering the Principal Curvatures and Visualization}

Not surprisingly, a direct classification of the principal curvatures (e.g. by a direct threshold) yields imperfect results, mainly because voxels are individually addressed. Consequently, an isolated point may be identified as a polyp. The 'neighbourhood' aspect is incorporated by clustering the curvature values as follows. First, the colon's interior volume is obtained by thresholding the image, followed by a region growing from the centerline. Then, a dilation of the resulting object is performed, with the restriction of 6-connectivity. The contour added contains the surface voxels. Clearly, only those voxels are of interest that have a principal radius of curvature within a certain range of values $\left(\mathrm{R}_{\text {low }}<\kappa_{1}<\mathrm{R}_{\text {up }}\right.$ and $\left.\mathrm{R}_{\text {low }}<\kappa_{2}<\mathrm{R}_{\text {up }}\right)$. Because such surface elements are expected to appear in clusters (due to the isotropy of polyps), a hierarchical clustering is performed by the single linkage method as described in [12]. At last, only those clusters are taken into account that contain a minimum number of voxels $\left(N_{\text {curvatures }}\right)$. The location of such a cluster is determined by projecting the centre of gravity into the closest point on the colon wall.

For visualization, we designed an environment presenting the bowel surface in a 'panoramic' way. The polyp detector has been integrated in this environment to support the radiologist.

Let us first look into some general aspects of standard colonography. Conventionally, the virtual colon is inspected by an animated image sequence from coecum to rectum and vice versa. Increasing the viewing angle enlarges the amount of surface inspected. However, this approach goes at the expense of increased distortions towards the edges of the image. To avoid extreme deformations while showing the full visible field around a position, we present the physician with a series of 'unfolded cubic renderings' [13]. 


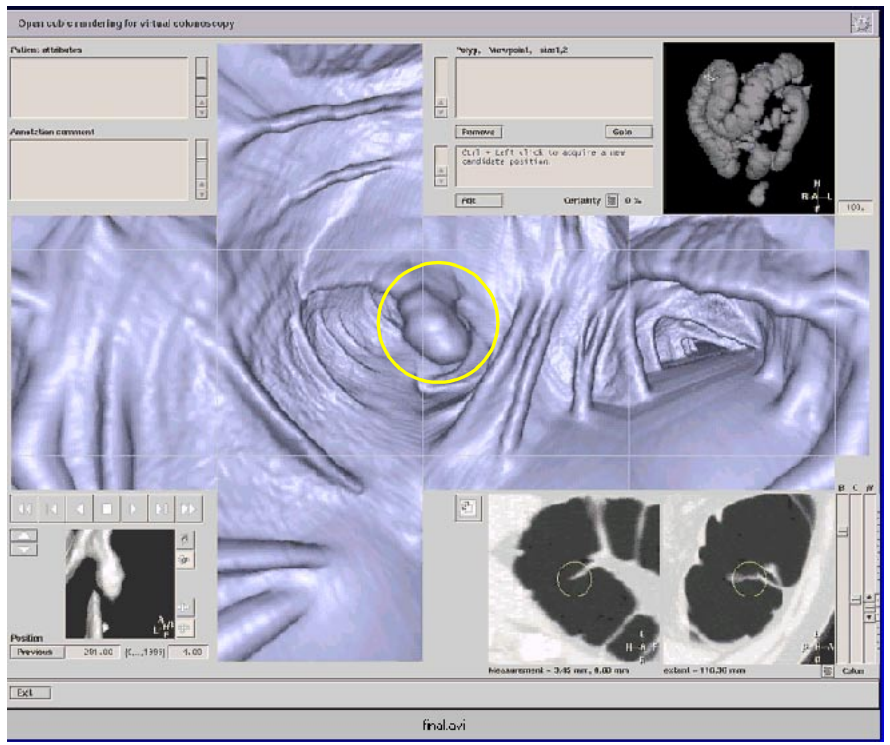

Fig. 2. A sequence of outfolded cubes is presented to improve efficiency

Consider a limited set of points sampled on the central path through the colon (for obtaining this central path we refer to [14]). Imagine that in each such position there is a cube of which the faces contain $90^{\circ}$ views from the centre. Clearly, projection of the six images onto a single plane (the 'unfolded cubes) renders the complete field of view (see Figure 2 for an example). Additionally, discontinuities are restricted to the edges.

The interface for reviewing the unfolded cube data is shown in Figure 2. In the central part a sequence of images is displayed. The overview image depicts the current global position in the colon (top right). Two reformatted views allow exploration of the original CT data (bottom right). Both images are generated automatically when the user clicks in the unfolded cube. The centre of the two images is determined by projecting the selected point on the colon wall. The first image is perpendicular to the path where it is closest to the selected position. The second, orthogonal cross-section is parallel to the centerline tangent. The physician can scroll interactively through these images and rotate them for closer inspection of suspicious areas.

The top-left window contains a list of detected polyps. By clicking on any one item, the unfolded cube image is shown, rendered from the closest central path point. The position of such a polyp is always indicated by a circle.

\section{Results}

This section describes an evaluation of our approach, specifically in relation to requirements of scale dependency, sensitivity and effectivity (surface in view). 


\subsection{Calculation of the Principal Curvatures}

We will test the calculation of the principal curvatures by its accuracy and precision. Obviously, both will vary as a function of $\sigma_{\text {Gauss }}$ (width of the Gaussian derivatives, see Section 2.1). Clearly, $\sigma_{\text {Gauss }}$ must be tuned to the size of the object under consideration (radius) and the noise level. At a typical CT image resolution of $0.5 \mathrm{~mm} /$ pixel, relevant polyps of 5-10 $\mathrm{mm}$ in diameter are approximated by spheres of 5 to 10 pixels in radius. In our CT images (see Section 3.2) we measured a contrast to noise ratio of about 0.05 . To monitor the effect of object size and noise we conducted the following experiments. A sphere with radius 5 pixels was generated (in the Fourier domain to obtain bandlimitedness). Those points were selected that were within 1 pixel from the true surface. Thus, the latter distance corresponds to positions 4 to 6 pixels from the centre. For each of these surface points the principal radii of curvature were calculated. The true values (gold standard) are given by the distance to the centre of the sphere. Consequently, the mean as well as the standard deviation were determined of the difference of ground truth and measured radius of Gaussian curvature $\left(=\kappa_{1} \cdot \kappa_{2}\right)$. Each of these two numbers (the 'bias' and standard deviation) were attained for varying $\sigma_{\text {Gauss }}$ and an array of noise levels (Gaussian). The graphs in Figure 3 show normalized results (i.e. the outcome divided by the squared true radius).

As expected, a certain minimal filter width $\left(\sigma_{\text {Gauss }}=3-5\right.$ pixels $)$ is required to obtain good accuracy and precision at any noise level. From the 'bias' it appears that the Gaussian curvature is increasingly overrated (note that the curves show gold standard - measured Gaussian curvature). Because the measurements involve a spherical object, the area outside the sphere relatively increases with larger $\sigma$. Consequently, the outcome is weighted with larger radii of curvature, yielding a biased result.

Previously (Section 1) we remarked that a distinguishing feature of polyps is that they jut out into the colon. While the plicae semilunares (or haustral folds, visible in Figure 1) have a protruding character in one principal direction, they have opposite curvature in the other. Because polyps present an smaller image structure than the colon surface, we propose to use the largest sigma that allows good measurement. Thus, to measure a polyp of $0.5 \mathrm{~cm}$ (diameter) at a resolution of $0.5 \mathrm{~mm} /$ pixel requires $\sigma_{\text {Gauss }}=5-9$ (compare with Figure 3). In this way such a clinically relevant polyp is measured with good accuracy and precision. On the other hand irrelevant features (smaller protrusions) will be smoothed away as much as possible into the background (having opposite curvature).

\subsection{Clustering and Visualization}

For the evaluation of our work we collected data from 20 patients visiting the Academic Medical Centre Amsterdam. An informed-consent procedure was followed, in which the patient inclusion criterion was the referral for conventional colonoscopy. For each patient 3D image data were acquired by computed tomography (CT) in supine and prone position. The in-plane resolution of the images was approximately $0.6 \mathrm{~mm} / \mathrm{pixel}$ at 

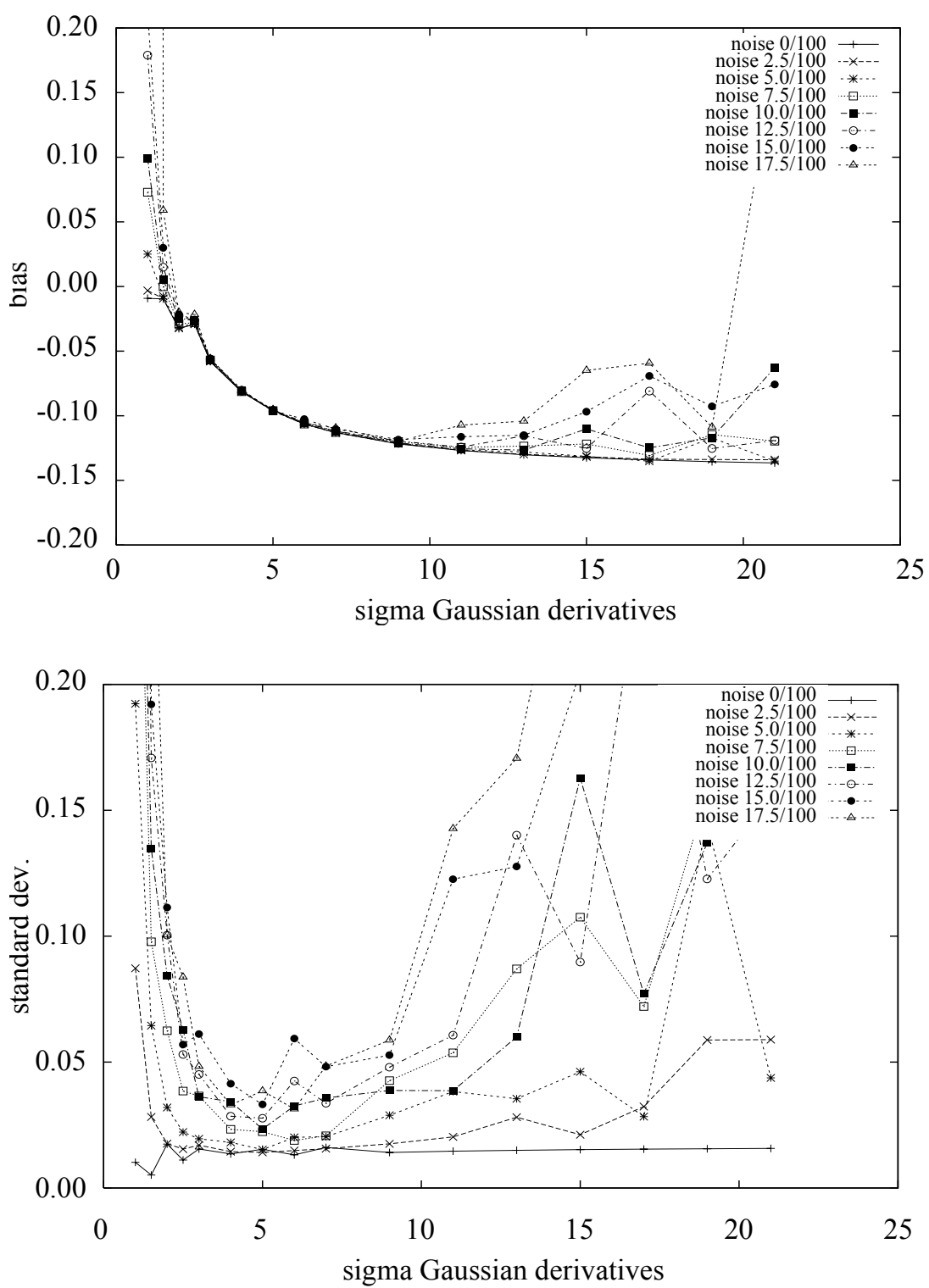

Fig. 3. A sphere with radius 5 pixels was generated. Those points were selected that were within 1 pixel from the true surface (i.e. 4 to 6 pixels from the centre). For each of these surface points the principal radii of curvature were calculated. The true values (gold standard) are given by the distance to the centre of the sphere. For all these points the mean as well as the standard deviation were determined of the difference of ground truth and measured radius of Gaussian curvature (= $\kappa_{1} \cdot \kappa_{2}$ ). Each of these two numbers (the 'bias' and standard deviation) were attained for varying $\sigma_{\text {Gauss }}$ and an array of noise levels. The graphs show normalized results 
a slice thickness of $3.2 \mathrm{~mm}$. The reconstruction interval was $1.6 \mathrm{~mm}$, resulting in half overlapping slices.

Each patient subsequently underwent optical colonoscopy. Identified polyps were resected and their size, location and morphology were annotated to define the ground truth. In 10 patients 15 colonic polyps were identified. Unfortunately, only 6 polyps were larger than $5 \mathrm{~mm}$. The interface (described previously) was implemented on an experimentally enhanced version of the EasyVision Endo-3D software package (Philips Medical Systems).

From Section 2 it can be deduced that our polyp detection comes with parameters $\sigma_{\text {Gauss }}, \mathrm{R}_{\text {low }}, \mathrm{R}_{\text {up }}$, and $N_{\text {curvatures }}$. Pursuing Section 3.1 we opt for $\sigma_{\text {Gauss }}=7.0$ in $\mathrm{x}$ and $\mathrm{y}$ and $\sigma_{\text {gauss }}=2.6(=7 /(1.6 / 0.6))$ in the z-direction to cope with the anisotropy.

$\mathrm{R}_{\text {low }}$ and $\mathrm{R}_{\text {up }}$ are introduced to obtain a tentative voxel classification. These parameters affect the outcome in the next manner. Ideally, the breaking point to select surface patches protruding into the colon lies around $\kappa_{1}, \kappa_{2}=0.0$. It will not come as a surprise that the number of falsely selected radii decreases rapidly with increasing $\mathrm{R}_{\text {low }}$. However, if this parameter is set too large, then small polyps may be excluded. Also, it turns out that radii near the apex are rejected from larger polyps.

Too small $\mathrm{R}_{\text {up }}$ (e.g. $10 \mathrm{~mm}$ ) appears to suppress features near the stem of the polyp first. Above approximately $20 \mathrm{~mm}$ this threshold hardly affects the outcome. Only extremely large values $\left(\mathrm{R}_{\mathrm{up}}=100 \mathrm{~mm}\right)$ result in selection of some voxels on haustral folds. Modelling the expected shape of polyps, we will apply $R_{\text {low }}=1.0 \mathrm{~mm}$ and $R_{u p}=20 \mathrm{~mm}$. Any remaining false positive radii are rejected by a constraint on the number of voxels that a cluster of them must contain $\left(N_{\text {curvatures }}\right)$. It was experimentally determined that a sphere of $10 \mathrm{~mm}$ in diameter consists of approximately 500 voxels (at a resolution of $0.6^{2} \times 1.6 \mathrm{~mm} /$ sample). In concordance we will use $N_{\text {curvatures }}=250$. The latter choice may seem arbitrary. However, changing the parameter by several decades did not appear to influence the outcome. Application of the aforementioned values resulted in detection of 5 out of 6 polyps ( $>5 \mathrm{~mm}$ ). The false positively detected lesions ranged from 5-15 per image volume. The false negative polyp is best described as a faintly sloped mound (see Figure 4). Clearly, our polyp detector is not designed to track down such shapes. Still, however, as the type of polyp is regarded significant, we need to adjust our method. To that end, we consider scale adaptive filtering.
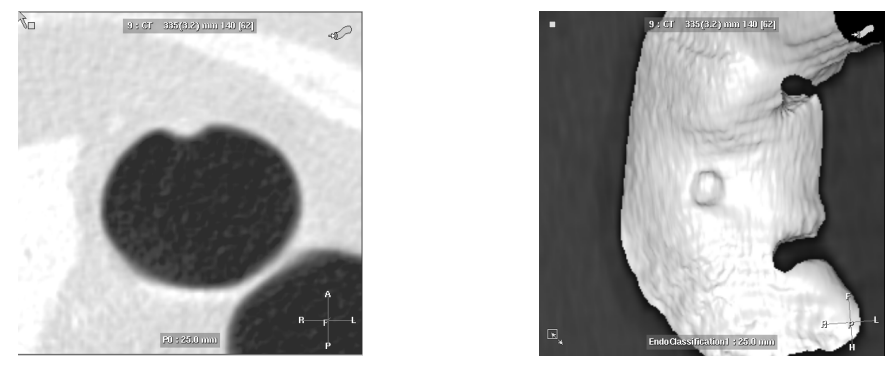

Fig. 4. An undetected polyp, on the left depicted in a reformatted CT image, on the right the virtual endoscopic view. 
The effectiveness of the visualization was tested by calculating the surface area coming into view. Therefore, the wall voxels in each volume were identified in the same manner as described earlier (Section 2.2). Subsequently, it was determined which fraction of them was projected into the images upon navigation along the central path (as in [15]). The results are summarized in Table 1 . The single plane entry is defined by considering the backward and forward views individually $(\mathrm{n}=80)$. In a two sided view (the conventional approach) both sequences are considered simultaneously $(n=40)$.

\begin{tabular}{|c|c|c|c|}
\hline Technique: & $\begin{array}{c}\text { Single plane } \\
\text { view }\end{array}$ & $\begin{array}{c}\text { Two sided } \\
\text { view }\end{array}$ & $\begin{array}{c}\text { Unfolded } \\
\text { cube }\end{array}$ \\
\hline \hline $\begin{array}{c}\text { Surface in view (+ } \\
\text { std. dev.): }\end{array}$ & $73 \%(2.8)$ & $93.8(0.5)$ & $99.5(0.1)$ \\
\hline
\end{tabular}

Table 1: Effectiveness in terms of surface in view.

The $6.2 \%$ of the total area not seen using the two sided view corresponds to about 20000 voxels, the largest cluster of which contained 1200 elements. This is significantly more than the surface of a polyp, which is approximately 500 voxels (see above). The latter discrepancy clearly justifies the unfolded cube visualization.

The sensitivity and specificity (on a per patient basis) are collated in Table 2. The gold standard was obtained through retrospective examination of the image sets by one radiologist specialized in CT colonography. The evaluation times were 28 minutes for the

\begin{tabular}{|c|c|c|}
\hline Technique: & $\begin{array}{c}\text { Two sided } \\
\text { view }\end{array}$ & $\begin{array}{c}\text { Unfolded } \\
\text { cube }\end{array}$ \\
\hline \hline Sensitivity (\%): & $7 / 10$ & $10 / 10$ \\
\hline Specificity (\%): & $8 / 10$ & $7 / 10$ \\
\hline
\end{tabular}

Table 2: Sensitivity and specificity for the conventional and unfolded cube approaches.

two sided view technique and 12 minutes using the unfolded cubes (see also [13]. For the review, the 'conventional' virtual endoscopy system as well as the unfolded cube technique were used both supported by automatic polyp detection. As expected (due to the larger field of view), more polyps were retrieved using the outfolded cube than with the conventional approach (two sided view). Concurrently, however, the number of false positives increases, yielding slightly lower specificity. We acknowledge that the low number of cases does not reveal significant differences. Clearly, further study is required in this respect.

\section{Conclusions}

A modern technique to screen for colorectal polyps is by virtual endoscopy. Any such visualization relies heavily on a proper representation of the geometric information. For optimal exploitation of surface geometry, automatic detection of polyps can serve to assist the radiologist. We presented a method based on the local principal curvatures. It was shown that all round polyps could be detected at the expense of a limited number 
of false positives. However, flat and elongated specimens require a more sophisticated approach, e.g. by adaptive filtering. Still, we consider automatic detection a promising support for inspection.

The unfolded cube visualization is another step to optimize polyp detection by visual examination. The method imposes that the complete colon surface comes into view. The experiments showed a sensitivity of $10 / 10$ for significant polyps.

Virtual endoscopy is rapidly becoming a state-of-the art tool for radiologists. The methods described in this paper aimed to contribute to its development.

\section{References}

[1] B. Vogelstein, E.R. Fearon, S.R. Hamilton, 'Genetic alterations during colorectal-tumour development', N. Engl. J. Med., vol. 319, pp. 525-532, 1988.

[2] J.D. Potter, M.L. Slattery, R.M. Bostick, 'Colon cancer: a review of the epidemiology', Epidemiol. Rev., vol. 15, pp. 499-545, 1993.

[3] J.G. Fletcher, W. Luboldt, 'CT colonography and MR colonography: current status, research directions and comparison', Eur.Radiol., vol. 10, pp. 786-801, 2000.

[4] N.W. Toribrara, M.H. Sleisenger, 'Screening for colorectal cancer', $N$ Engl J Med, vol. 332, pp. 332, pp. 861-867, 1995.

[5] G.D.Dodd, 'Colon cancer and polyps imaging perspectives', Proc. First International Symposium on Virtual Colonoscopy, pp. 15-17, Boston University Press, 1998.

[6] D.K. Rex, C.S. Cutler, G.T. Lemmel, 'Colonoscopy miss rates of adenomas determined by back-to-back colonooscopies’, in Gastroenterology, vol. 112, pp. 24-28, 1997.

[7] S.J. Winawer, R.H. Fletcher, L. Miller, 'Colorectal cancer screening: clinical guidelines and rationale', Gastroenterology, vol. 112, pp. 594-642, 1997.

[8] L. Hong, S.Muraki, A. Kaufman, T. He, 'Virtual voyage: interactive navigation in the human colon', Proc. ACM SIGGRAPH Conf., pp. 27-34, ACM Press, 1997.

[9] C.F. Beaulieu, 'Computer aided detection of colonic polyps', Second International Symposium on Virtual Colonoscopy, pp. 73-77, Boston University Press, 2000.

[10] R.M. Summers, C.F. Beaulieu, S.Napel, 'Automatic polyp detector for CT colonography: feasibility study', Radiology, vol. 216, pp. 284-290, 2000.

[11] L.J. van Vliet and P.W. Verbeek, 'Curvature and bending energy in digitized 2D and 3D images', Proc. 8th Scandinavian Conf. on Image Analysis, pp. 1403- 1410, 1993.

[12] M.R. Anderberg, 'Cluster analysis for applications', Academic Press Inc., 1973.

[13] I.W.O. Serlie, F.M. Vos, R. van Gelder et al. 'Improved visualization in virtual colonoscopy using image-based rendering', accepted for publication in Proc. ACM/Eurographics Vis Sym, 2001.

[14] R.Truyen, P.Lefere, S.Gryspeerdt, T.Deschamps, 'Speed and robustness of (semi-) automatic path tracking', Proc. Second International Symposium on Virtual Colonoscopy, p. 102, 2000.

[15] D.S. Paik, C.F. Beaulieu, R.B. Jeffrey, C.A.. Karadi, S. Napel, 'Visualization modes for CT colonography using Cylindrical and planar map projections', J Comput Assist Tomogr., vol. 24(2), pp. 179-188, 2000. 